\title{
Comparative histopathological and immunological study of two field strains of chicken anemia virus
}

\author{
Agustina Rimondi ${ }^{1,3^{*}}$, Silvina Pinto ${ }^{2}$, Valeria Olivera ${ }^{1}$, Marina Dibárbora ${ }^{1,3}$, Mariano Pérez-Filgueira ${ }^{1,3}$,
} María Isabel Craig ${ }^{1}$ and Ariel Pereda ${ }^{1,3}$

\begin{abstract}
Infection of poultry with chicken anemia virus (CAV) is implicated in several field problems in broiler flocks due to the immunosuppression generated and, consequently, the increased susceptibility to secondary infections. Recently, we have reported an increased occurrence of clinical cases caused by CAV strains distantly related to those commonly used for vaccination. In order to understand the behavior of two Argentinean CAV strains (CAV-10 and (AV-18) in two-week-old chickens, an immune and histopathological study was performed. Neither mortality nor clinical signs were observed in the infected or control groups. Thymus lobes from chickens infected with both CAV viruses were smaller compared to the negative control group. At 14 days post-infection (dpi), only chickens inoculated with CAV-10 show a severe depletion of lymphocytes in the thymus cortex and in follicles from the bursa of Fabricius. Also thymopoiesis disorders, such as reduction in the percentage of total DP (CD4 + CD8a+) thymocytes and alteration in the percentages of DP subpopulations, were more important in animals inoculated with the CAV-10 than the CAV-18 strain. In addition, only animals infected with CAV-10 show a decrease in CD8a $\beta$ splenocytes. Altogether our results show that, although both Argentinean CAV strains produce subclinical infections in chickens causing immunosuppression at 14 dpi, they might differ in their in vivo pathogenicity.
\end{abstract}

\section{Introduction}

Chicken anemia virus (CAV) is a closed circular negative single-stranded DNA virus [1] from the Circoviridae family that belongs to the Gyrovirus genus [2]. The virus genome encodes a polycistronic mRNA from which three viral proteins are translated [3,4]: ORF1 encodes VP1, the only capsid protein; ORF2 encodes VP2, a scaffold protein which allows the proper folding of VP1 [5,6]; and ORF3 encodes VP3, which is known as an apoptin that induces apoptosis of thymocytes after in vivo infection and apoptosis of transformed avian cell lines after in vitro infection $[7,8]$.

\footnotetext{
* Correspondence: rimondi.agustina@inta.gob.ar

${ }^{1}$ Laboratorio de Aves y Porcinos, Instituto de Virología CICVyA, Instituto Nacional de Tecnología Agropecuaria (INTA), CC25, B1712WAA Castelar, Buenos Aires, Argentina

${ }^{3}$ Consejo Nacional de Investigaciones Científicas y Tecnológicas (CONICET), Rivadavia 1917, C1033AAJ CABA, Argentina

Full list of author information is available at the end of the article
}

Worldwide, CAV causes considerable economic losses in the poultry industry because it is responsible for a clinical disease with high mortality characterized by anemia, haemorrhages and atrophy of the thymus and bone marrow in young chicks [9]. Infection of chickens older than two weeks of age, although considered subclinical, also has immunosuppressive effects with important consequences on growth and profitability of poultry [10-12]. Markowsky-Grimsrud and Schat [13] have demonstrated that experimental infection with CAV also impairs the generation of pathogen-specific cytotoxic T lymphocytes (CTL) that may have important implications for adaptive cell-mediated immunity to several secondary pathogens. This was also observed by other authors that reported secondary infections with other viruses, bacteria, parasites and/or fungi [1,14-16].

Experimental infection of chickens with CAV induced a substantial but transient decrease in some immunological parameters corresponding to a generalized lymphocytopenia 
in the thymus, bursa and spleen [17-19], with a peak at 14 days post-inoculation (dpi) and complete recovery at $28 \mathrm{dpi}$. These observations were observed in 1 or 14-dayold inoculated chickens $[19,20]$. While there is a consensus on the idea that absolute numbers of thymic lymphocytes dramatically decrease during the period of transient leukopenia [19-21], the selective alterations in the percentages of $\mathrm{T}$ lymphocyte subpopulations in the spleen during the acute phase is still under discussion. Coud et al. [19] showed a greater depletion of CTL than T-helper lymphocytes (ThL) in the spleen at $14 \mathrm{dpi}$, while $\mathrm{Hu}$ et al. [20] showed no selective decrease of ThL or CTL by flow cytometric analysis of CD4+ and CD8+ subpopulations. On the contrary, Vaziry et al. [21] showed a reduction in the percentage of CD4+ splenocytes only at 7 and $28 \mathrm{dpv}$, with a simultaneous increase in the percentages of CD8+ cells. It is noteworthy that most experimental infections with CAV have been done with reference strains such as CIA-1 or Cux-1 [19-24]; therefore, knowledge of the pathogenicity of CAV field strains remains poor and needs to be studied more.

In 2009, our group reported the circulation of CAV affecting both broilers and layers in commercial flocks in Argentina [25]. Sequence analysis of predicted VP1 peptides showed that most of the Argentinean viruses have a glutamine residue at positions 139 and 144, suggesting that these viruses might have a reduced spread in an avian lymphoblastoid cell line (MDCC-MSB1) compared with Cux-1 [26]. We showed that the circulation of CAV in commercial poultry in Argentina clearly affect their immune status through a subclinical infection [25]. Therefore, it would be important to broaden and deepen our knowledge about the effect of subclinical experimental infections with Argentinean CAV strains in chickens, moreover considering the high frequency of clinical cases and despite the fact that vaccination against CAV is common in breeder flocks in Argentina.

The present study addresses the effect of subclinical infection with two Argentinean CAV strains at $14 \mathrm{dpi}$. We studied the percentage of T-lymphocyte subpopulations in the thymus and spleen, and the histopathological changes produced in the thymus and bursa of Fabricius by both viruses. In addition, this paper contains the first two complete genome sequences of CAV published from Argentinean field strains and the first report of the behaviour of these viruses in vivo.

\section{Materials and methods}

\section{Amplification and sequencing of the CAV genome}

Firstly, a pair of primers was designed to obtain the complete genome of CAV (Table 1): CAV-NheI Fw and CAV-NheI Rv. The primers were used under cycling conditions of denaturation $\left(5 \mathrm{~min} 95^{\circ} \mathrm{C}\right)$, followed by $35 \mathrm{cy}$ cles of denaturation $\left(30 \mathrm{~s} 95^{\circ} \mathrm{C}\right)$, annealing $\left(30 \mathrm{~s} 54^{\circ} \mathrm{C}\right)$ and extension $\left(5 \mathrm{~min} 72{ }^{\circ} \mathrm{C}\right)$. After a final extension $(10 \mathrm{~min}$ $72{ }^{\circ} \mathrm{C}$ ), samples were held at $16{ }^{\circ} \mathrm{C}$, until visualization in a $1 \%$ agarose gel with ethidium bromide.

PCR products were purified with a QIAquick PCR purification kit (Qiagen) and sequencing was performed using the BigDye Terminator v3.1 Cycle Sequencing Kit on an ABI PRISM 3700 DNA Analyzer (Applied Biosystems) according to the manufacturer's instructions. Sequences were carried out with the same amplification primers and internal primers CAV1, CAV2, CAV-VP1-A fw, CAVVP1-A rev, CAV-VP1-B fw and CAV-VP1-B rev previously described [25,27]. Six new additional internal primers were designed to obtain the complete CAV genome through overlapping fragments: CAV-Genome Fw, CAV-Genome Rv, CAV-VP2 Fw, CAV-VP3 Rv, CAV-Sec1 Rv and CAV-Sec4 Fw (Table 1).

The consensus amino acid and nucleotide sequences for both CAV genomes were generated using Megalign (DNASTAR, Madison, WI, USA). Sequences were assembled and edited with Lasergene 8.1 (DNASTAR); BioEdit 7 was used for alignment and residue analysis.

\section{Chickens and housing}

Specific-pathogen-free (SPF) White Leghorn embryonated eggs free of CAV antibodies were purchased (Rosenbusch S.A. CABA, Argentina), incubated and hatched in an automatic incubator (Yonar, CABA, Argentina) under appropriate conditions, and randomly separated into

Table 1 Sequences of the primers used to obtain the complete genome sequence of CAV strains

\begin{tabular}{|c|c|c|c|}
\hline Oligo name & Sequence & Length & Position $^{a}$ \\
\hline CAV-Nhel Fw & 5'-GCTAGCGTCAATGAACCTGA-3' & 20-mer & $1191-1210$ \\
\hline CAV-Nhel Rv & 5'-GCTAGCAGGAACTCTTTCA-3' & 19-mer & 1178-1196 \\
\hline CAV-Genome Fw & 5'-ACGCTAAGATCTGCAACT-3' & 18-mer & $651-668$ \\
\hline CAV-Genome Rv & 5'-GTAATTCCAGCGATACCAA-3' & 19-mer & $589-607$ \\
\hline CAV-VP2 FW & 5'-ATGCACGGGAACGGCGGACAACC-3' & 23-mer & $380-402$ \\
\hline CAV-VP3 RV & 5'- TTACAGTCTTATACACCTTCTTGCGGTTCG-3' & 30-mer & $822-851$ \\
\hline CAV-Sec1 Rv & 5'-AACGTCACTTTCGCAACGT-3' & 19-mer & $278-296$ \\
\hline CAV-Sec4 Fw & 5'-AGGGCAACGTTCAGTTTCTA-3' & 20-mer & 1833-1852 \\
\hline
\end{tabular}

${ }^{\mathrm{a} G e n B a n k}$ accession no. M55918. 
three groups of 6 chickens. Groups were housed separately in sterilized isolators for chickens under negative pressure conditions (Allentown CH8ISOL) with food and water ad libitum throughout the experimental period. Animal care and experimental procedures were performed in accordance with the approved protocols of the National Institute of Agricultural Technology Ethics Committee (INTA, Argentina).

\section{Viruses and inocula}

The Argentinean CAV-10 and CAV-18 viruses were obtained from commercial broilers in 2007 with increase of mortality, gangrenous dermatitis and thymus and bursa atrophy [25]. These strains were unable to be grown in MDCC-MSB1 cells. Therefore, under our experimental conditions, inocula were adjusted to $10^{6}$ copies of CAV DNA per animal for comparative analyses of our results. Argentinean CAV viruses were propagated in chickens and CAV inocula were prepared from thymus samples as previously described [28]. Next, DNA was obtained with the QIAamp DNA Mini Kit (Qiagen Inc.Valencia, CA, USA) according to the manufacturer's instructions, and the number of CAV genomes contained in each inoculum was determined by SYBR Green-based real-time quantitative PCR (RT-qPCR). Values were converted to number of copies of CAV DNA per $\mu \mathrm{L}$ of inoculum. Finally, the inocula were adjusted to $1 \times 10^{6}$ copies of CAV DNA/200 $\mu \mathrm{L}$.

\section{Quantitative Real Time PCR}

SYBR Green-based quantitative real-time PCR (RT-qPCR) was used to determine the number of copies of the CAV genome in inocula.

\section{Reagents}

CAV DNA was amplified using reagents and primers purchased from Biodynamics. (CABA, Argentina). Primers used amplify a fragment (75 bp) located within the overlapping region between ORF2 and ORF3 (Table 2). The primers were designed and evaluated using Primer Express v1.0 software. Individual PCR reactions, containing 15 pmol of each primer in a total volume of $25 \mu \mathrm{L}$, were performed in an ABI Prism 7500 SDS system (Applied Biosystems, Warrington, UK).

\section{Cycling parameters}

The cycling parameters consisted of the following steps: $95^{\circ} \mathrm{C}$ hold for $10 \mathrm{~min}$, followed by 40 cycles consisting of denaturation at $95{ }^{\circ} \mathrm{C}$ for $15 \mathrm{~s}$ and annealing/extension at
$50{ }^{\circ} \mathrm{C}$ for $1 \mathrm{~min}$, and finally adding a dissociation melting curve analysis.

\section{Preparation of standards}

A fragment of $75 \mathrm{bp}$ of the CAV genome from Cux-1 vaccine strain was amplified by PCR using the primers described in Table 2 and cloned in pGEM T-Easy vector (Promega Madison, USA) to generate standard curves for the quantitation of number of copies of CAV DNA. Fresh tenfold dilutions were made from the plasmid stocks in distilled water for each PCR run to avoid the problem of plasmid degradation over time in the higher dilutions. Cycle threshold (CT) values were used to plot a standard curve and a range from $10^{1}$ to $10^{6}$ copy numbers was represented in each standard curve. The copy number was determined using the conversion factor: 1 ug of a $1-\mathrm{kb}$ DNA $=3.03$ pmol ends [29].

\section{Analysis}

The results from the PCR experiments were analyzed using Sequence Detection Systems v.1.6.3 software. The default settings of the program were used to define both the threshold value and baseline parameters for analysis of the raw data. A standard curve was generated in each assay and used to extrapolate the amounts in the unknown samples.

\section{Experimental design}

Six two-week-old chickens were intramuscularly inoculated with $200 \mu \mathrm{L}$ of CAV-10 or CAV-18 containing $1 \times$ $10^{6}$ CAV DNA copies. Negative control group $(n=6)$ was inoculated with $200 \mu \mathrm{L}$ of phosphate-buffered-saline (PBS). Signs of anorexia, weakness, stunting, cyanosis, petechiae and ecchymoses were looked for everyday in every bird. Fourteen days after inoculation, chickens were bled, euthanized and body, thymus, spleen and bursa of Fabricius were weighed and recorded in all birds. From each animal, the bursa and the first right lobe of the thymus were separated for histopathological evaluation. Fractions of the thymus and spleen were also kept for flow cytometry evaluation.

\section{Histopathology}

Samples of the thymus and bursa of Fabricius were collected and immersed into 10\% neutral buffered formalin for fixation. Samples were dehydrated and embedded in paraffin wax in the usual manner, sectioned ( $4 \mu \mathrm{m}$ thick) and stained with hematoxylin and eosin (HE).

Table 2 Primers used in quantitative real time PCR to detect and quantify chicken anemia virus DNA

\begin{tabular}{llll}
\hline Primer & Sequence & Length & Position $^{\text {a }}$ \\
\hline CAV-Real Time FW & 5'-AGAGAGATCCGGATTGGTATCG-3' & 22-mer & $576-597$ \\
CAV-Real Time Rv & 5'-TGGGAGCGCGAGCATT-3' & 16-mer & $636-651$ \\
\hline
\end{tabular}

${ }^{\mathrm{a}}$ GenBank accession no. M55918. 


\section{Lymphocyte isolation}

Single cell suspensions from spleens were obtained by direct mechanical disruption in RPMI 1640 through a $40 \mu \mathrm{m}$ mesh (Cell Strainer, BD). Thymuses were cut into very small pieces and mechanically disrupted by pressing with a syringe plunger, in RPMI 1640. Then, cellular suspensions were passed through a $40 \mu \mathrm{m}$ mesh (Cell Strainer, BD). Mononuclear cells were isolated from both suspensions by centrifugation over Histopaque density gradient (1.077 g/mL; Sigma, St. Louis, MO, USA) at $400 \times g$ for $30 \mathrm{~min}$ and room temperature. Cells were isolated from the interface, washed, and live cells were counted using trypan blue exclusion.

\section{Flow cytometry analysis}

Cells were diluted in staining buffer (PBS $1 \times, 10 \%$ FBS, $0.1 \%$ Sodium Azide) and $1 \times 10^{6}$ cells per well were transferred into 96 well-plates (V-shape), and washed twice with the same buffer. Staining was performed by resuspending the cellular pellet of each well with $100 \mu \mathrm{L}$ of staining buffer including different combinations of antibodies, or as single-color stainings for compensation. Cells were incubated at $4{ }^{\circ} \mathrm{C}$ for $30 \mathrm{~min}$ and washed twice with staining buffer by centrifugation at $250 \times g$ for $5 \mathrm{~min}$.

Avian monoclonal antibodies (mAbs) (CD3-SPRD, CD4-FITC, CD $8 \alpha-P E, C D 8 \alpha-F I T C$ and CD $8 \beta-P E)$ were purchased from Southern Biotech (Birmingham, AL, USA). Mononuclear cell suspensions from every thymus and spleen were stained with one and two mAb mix, respectively: CD3-SPRD, CD4-FITC and CD8 $\alpha$-PE in tube A (for thymocytes and splenocytes), and CD3-SPRD, CD $8 \alpha$-FITC and CD $8 \beta$-PE in tube B (splenocytes). All antibodies were titrated in order to determine the optimal staining concentration of each one.

Positive cells were analyzed with a FACS Calibur flow cytometer (BD Biosciences, San Jose, CA, USA) and CellQuest software. Analysis was done on 20000 events and discrete viable lymphoid cell populations were gated according to the forward/side scatter characteristics. Percentages of different lymphoid cell subpopulations in the thymus and spleen were determined by multiparametric analysis.

\section{Statistical analysis}

Data were tested for normal distribution prior to analysis (Shapiro-Wilk test) using the Statistical Package for the Social Sciences for Windows (SPSS, version 15.0, Chicago, IL, USA). An ANOVA test was used, and a Tukey's test was applied when differences were detected. Variables that did not fit the ANOVA's assumptions were analyzed by Kruskall-Wallis and Bonferroni-Dunn post hoc test. $P$-values of $p<0.05$ were considered statistically significant.

\section{Serology}

Sera were tested by competitive enzyme-linked immunosorbent assay (ELISA) for specific antibodies against CAV according to the manufacturer's recommendations (Idexx Laboratories, Inc., Westbrook, ME, USA) using 10-fold dilutions of each serum sample.

\section{Nucleotide sequence accession numbers}

The nucleotide genome sequence from Argentinean CAV strains obtained in this study are available from GenBank under accession numbers KJ872513 (CAV-10) and KJ872514 (CAV-18).

\section{Results}

\section{Characterization of CAV field strains used for} experimental infection of chickens

The complete nucleotide genome sequence of CAV-10 and CAV-18 was determined using primers described in Table 1. Both Argentinean CAV genomes are 2298 nt long, and lacked a 21-nt region located within the noncoding, transcription-regulatory region of the genome $[4,30]$ that is present in the Cux-1 isolate (M55918). A comparison between the two Argentinean CAV viruses shows 14 individual nucleotide sequence differences dispersed throughout the genome, with three being located in the non-coding region and the rest along ORF1. Nucleotide differences between VP1 from CAV-10 and CAV-18 resulted in only 2 amino acid changes, one Q294H and the other S370T. In addition, both viruses present a glutamine $(\mathrm{Q})$ residue at positions 139 and 144 of VP1.

\section{Clinical signs}

No mortalities occurred after CAV or PBS inoculation during the experiment. No clinical signs of disease and no differences in body weight were observed between CAV-inoculated and negative control groups.

\section{Macroscopic alterations}

Chickens inoculated with CAV-10 or CAV-18 show reddened and atrophied thymic lobes distributed on both sides along the neck. In contrast, the control group had whitish and firm thymic lobes (Figure 1). There were no other significant gross lesions in any of the groups.

Mean thymus-to-body weight ratios were reduced $(p<0.05)$ in both CAV-inoculated groups in comparison to the negative control group (Figure 2). In addition, these reductions were significantly different between CAV-10 and CAV-18 inoculated chickens. Spleens and bursas did not significantly alter their weight at this time postinfection.

Lymphocytes from mononuclear single cell suspensions from thymuses and spleens were counted using trypan blue exclusion, and cell depletion, expressed as the ratio 
a

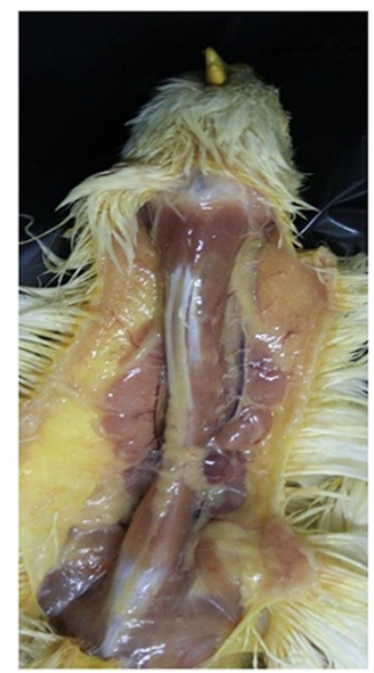

b

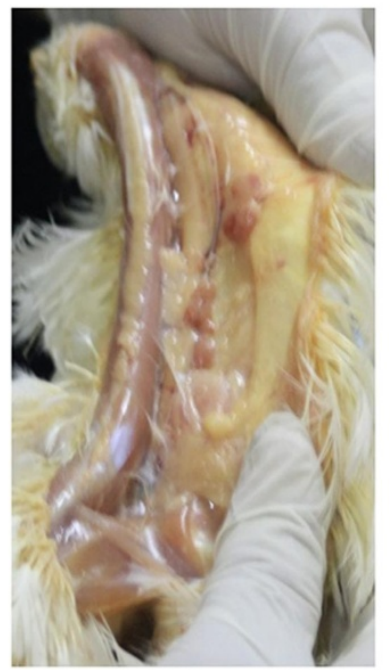

C

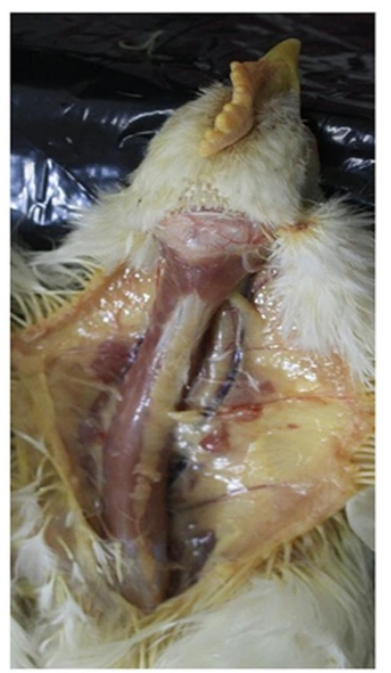

Figure 1 Gross lesions after 14 days post-infection with Argentinean CAV strains in 2-week-old chickens. Note that the thymic lobules from CAV-10 (b) and CAV-18 (c) inoculated chickens are smaller and reddened than those from the control group (a).

between the number of cells per weight of the organ, was analyzed in both organs. A significant reduction of cell counts was observed in the thymuses from chickens inoculated with CAV strains as shown in Figure 3 $(p<0.05)$.

\section{Histopathological changes}

On the one hand, thymuses from chickens inoculated with CAV-10 experienced severe lymphocyte depletion in the cortex (Figure 4b), showing a reduced demarcation between the medulla and cortex. Large lymphoblasts with karyomegaly and vacuolization of reticular cells were also observed in thymuses from CAV-10 inoculated chickens, producing a starry-sky appearance. Moreover, atrophy with moderate lymphoid depletion of the lymphoid follicles was observed in bursae from birds inoculated with CAV-10 (Figure 4e). Also, macrophage and plasma cell infiltration were observed in some of these bursae. On the other hand, thymus and bursa from CAV-18 inoculated chickens did not show histopathological changes at $14 \mathrm{dpi}$.

\section{Analysis of thymic cell subpopulations}

To verify whether Argentinean CAV strains induce disorders in lymphoid cell populations or not, the percentages of different lymphoid subsets in thymuses from CAV-inoculated chickens were compared to those from control birds in gate 1 (G1) according to forward/side scatter parameters. Thymocytes in G1 were divided into small (G2) and large (G3) cells to verify if thymopoiesis was also altered by CAV infection (Figure 5a).

Although the comparison of the lymphocyte percentages from each group shows no significant differences in G1, percentages in G2 and G3 were significantly different

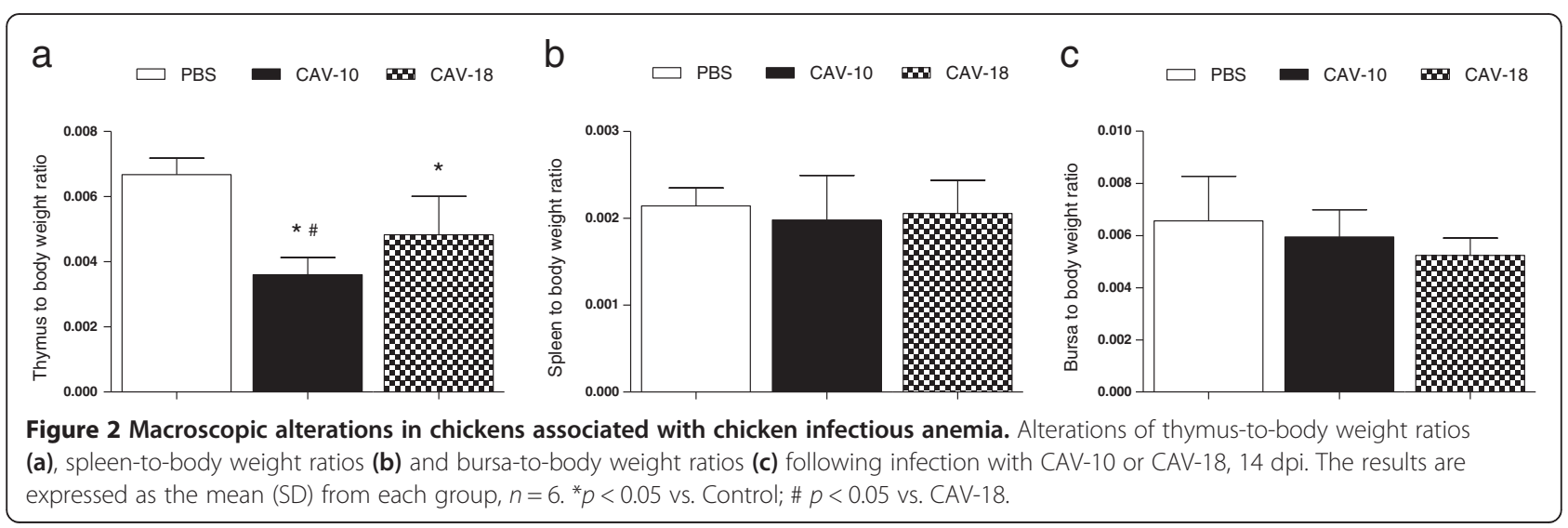



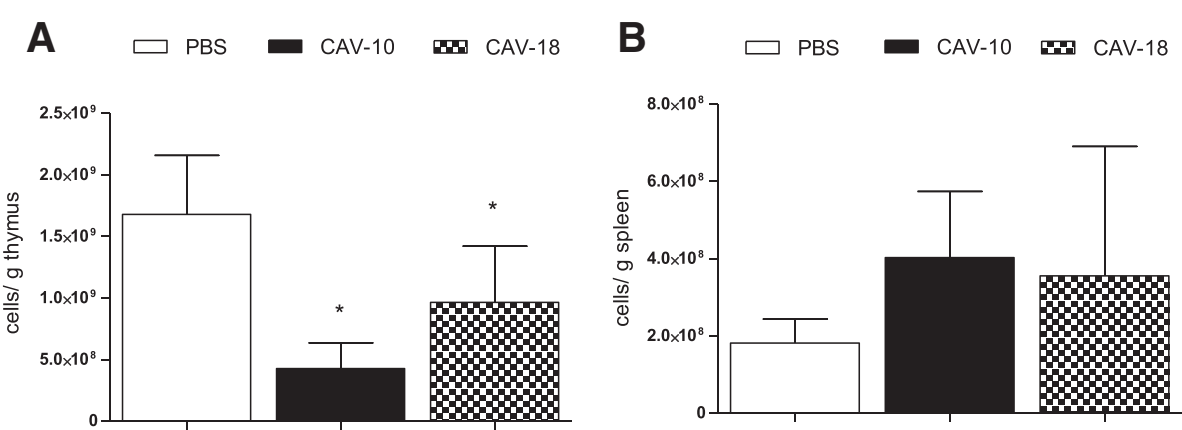

Figure 3 Lymphocyte depletion after infection with Argentinean CAV strains. Lymphocyte depletion expressed as the ratio of cells/g of thymus (A) or spleen (B) at 14 dpi in SPF chickens inoculated with CAV-10, CAV-18 or PBS at 2 weeks of age. The results are expressed as the mean (SD) from each group, $n=6 .{ }^{*} p<0.05$ vs. Control.

between groups (Figure 5b). There was a clear and important reduction in the percentages of lymphocytes in G2 from chickens inoculated with Argentinean CAV viruses $(p<0.05)$. In addition, CAV-10 show a higher reduction of lymphocytes in G2 than CAV-18 $(p<0.05)$. In contrast, the percentages of lymphocytes in G3 were increased in both CAV inoculated groups $(p<0.05)$. This increase was higher in thymuses from CAV-10 inoculated chickens with respect to the increase observed in thymuses from CAV-18 inoculated birds $(p<0.05)$.
The multiparametric analysis of $\mathrm{CD} 3+$ cells within the G1 region revealed the presence of CD4 + CD8 $\alpha-$, CD4$\mathrm{CD} 8 \alpha+$ and $\mathrm{CD} 4+\mathrm{CD} 8 \alpha+$ cell subsets. As shown in Figure $6 \mathrm{~A}$, the predominant subset in the control thymus was the double positive (DP) CD $4+C D 8 \alpha+$. Preliminary data show that these DP cells also express CD8 $\beta$ on their surface (data not shown), indicating that they are immature DP cells that did not undergo positive and negative selection, a characteristic during ontogeny of $\mathrm{T}$ lymphocytes. These DP cells in G1 were then divided into two
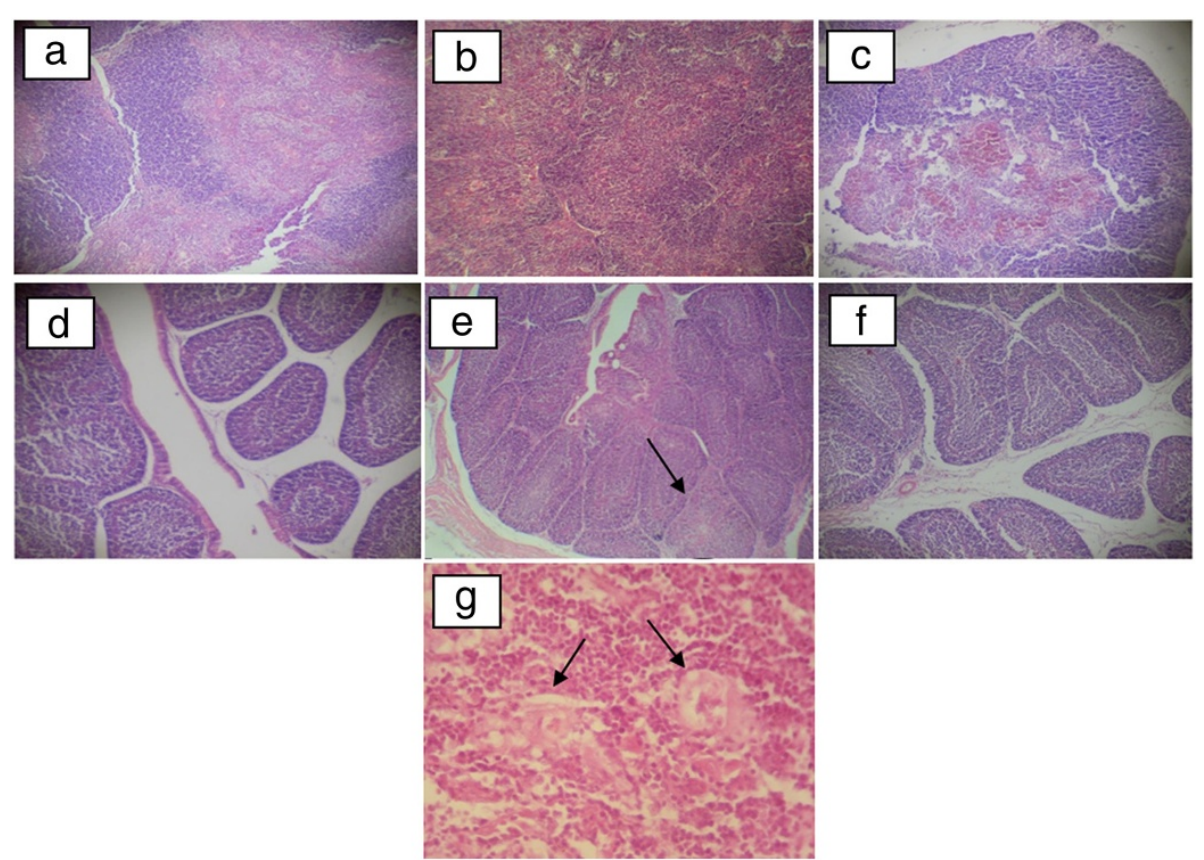

Figure 4 Histopathological findings of CAV infection in tissues from 4-week-old SPF chickens. Animals were studied 14 days after infection with CAV or inoculation with PBS at 2 weeks of age, and pictures show representative tissue samples from experimental and control groups stained with HE, 10× (a-f). (a) Thymus lobe from PBS inoculated group; (b) thymus lobe from CAV-10 inoculated group with severe cortical lymphocyte depletion and absence of demarcation between the medulla and cortex; (c) thymus lobe from CAV-18 inoculated group; (d) bursa of Fabricius from the PBS inoculated group; (e) bursa of Fabricius from CAV-10 inoculated group, showing moderate lymphoid depletion of the follicles with follicular atrophy marked with an arrow; (f) bursa of Fabricius from the CAV-18 inoculated group. (g) Large lymphoblasts with karyomegaly in thymuses from CAV-10 inoculated chickens, 40x; vacuolization of reticular cells were also marked by arrows. 


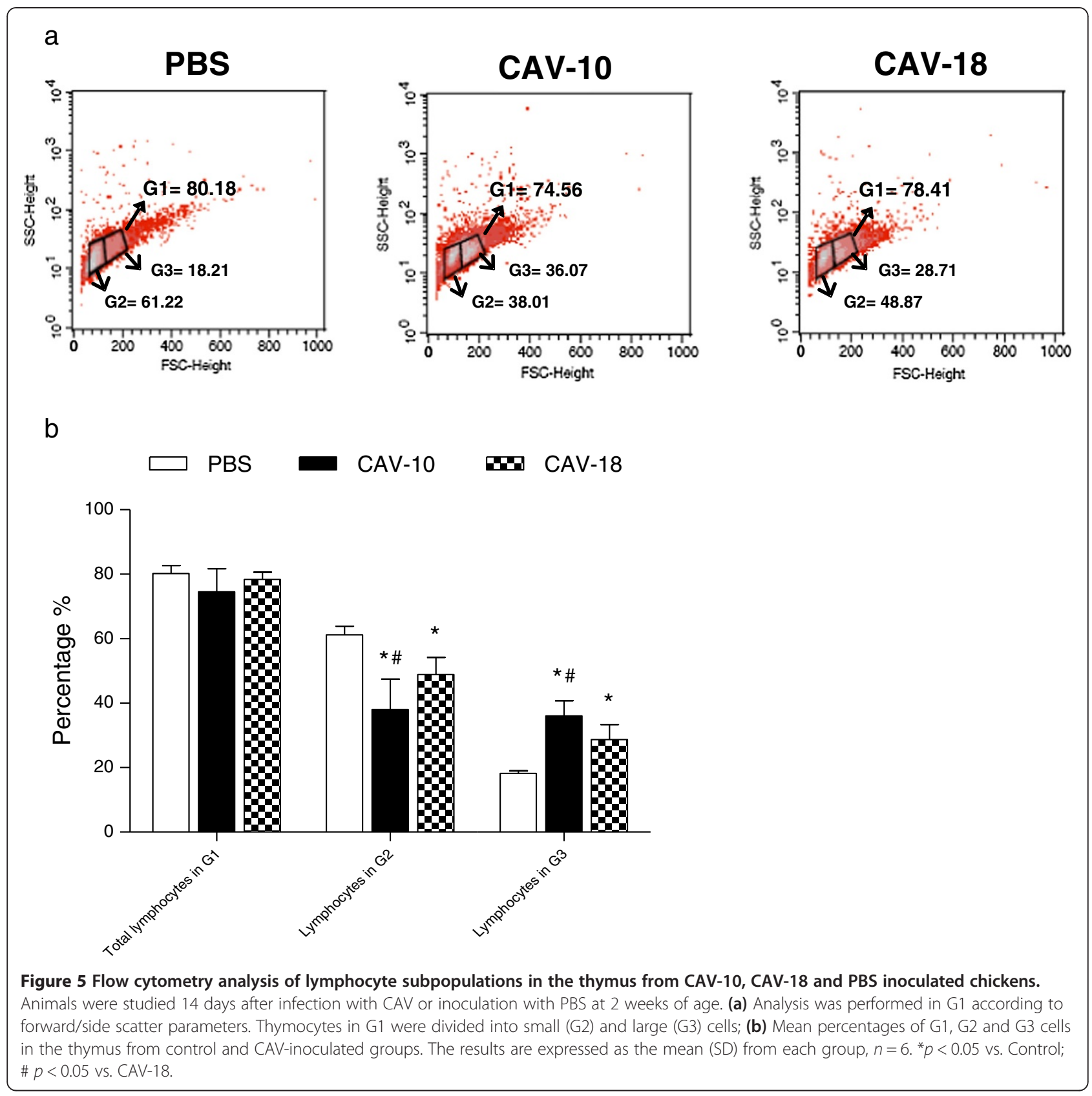

regions for further analysis, R4 and R5, according to the surface expression of CD4 and CD8 $\alpha$. While thymocytes in $\mathrm{R} 4$ have higher CD8 $\alpha$ expression than thymocytes in $\mathrm{R} 5$, thymocytes in R5 have higher CD4 expression than cells in R4.

Figures $6 \mathrm{~B}$ and $6 \mathrm{C}$ show DP cells from a thymus of the control group in G2 and G3 respectively. Most of the small DP with size and granularity compatible with G2 were in R4, whereas large DP cells from G3 were located in both $\mathrm{R} 4$ and $\mathrm{R} 5$. In particular, CAV-10 not only reduced $(p<0.05)$ the percentages of total DP thymocytes in G1, G2 and G3 as CAV-18 (Figure 6J-L), but also altered the percentage of DP thymocytes in R4 and R5 (Figure 6D-I). CAV-10 in particular produced a higher reduction of DP in R4 (in G1, G2 and G3) than CAV-18 $(p<0.05)$, and a particular significant increase of DP in R5 (G1 and G2) that was not seen in thymuses from CAV-18 inoculated chickens (Figure 6J-L).

Figures $6 \mathrm{~A}, 6 \mathrm{D}$ and $6 \mathrm{G}$ also show some differences in $\mathrm{CD} 4+\mathrm{CD} 8 \alpha-$ and CD4-CD8 $\alpha$ + thymocytes in G1 between control, CAV-10 and CAV-18 inoculated groups. Both CAV-inoculated groups appear to have increased their $\mathrm{CD} 4+\mathrm{CD} 8 \alpha-$ and $\mathrm{CD} 4-\mathrm{CD} 8 \alpha+$ subsets when relative percentages were analyzed; however, when the 


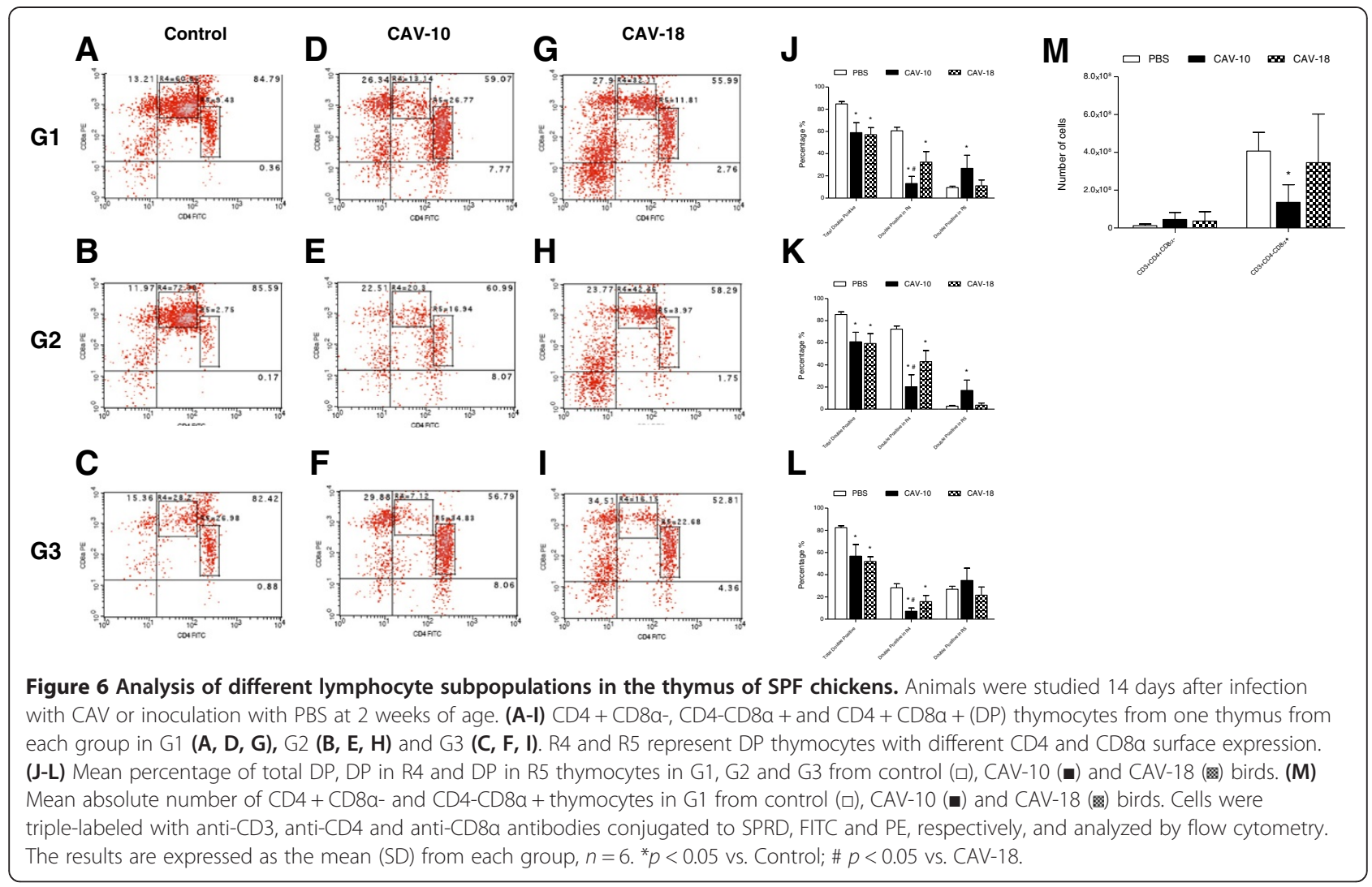

absolute number of CD $4+\mathrm{CD} 8 \alpha-$ and CD $4-C D 8 \alpha+$ thymocyte subpopulations were compared between groups, only the number of CD4-CD $8 \alpha+$ thymocytes were reduced in thymuses from CAV-10 inoculated chickens $(p<0.05)$ (Figure 6M).

\section{Analysis of splenic cell subpopulations}

Splenic lymphocytes from experimental groups were analyzed in G1 according to forward/side scatter parameters.

\section{Staining with $C D 3 / C D 4 / C D 8 a$}

The analysis revealed the presence of $\mathrm{CD} 3+\mathrm{CD} 4+$ CD $8 \alpha-($ ThL $), \quad C D 3+C D 4-C D 8 \alpha+(\mathrm{CTL})$ and $\mathrm{CD} 3+$ $\mathrm{CD} 4+\mathrm{CD} 8 \alpha+($ mature DP) cell subsets. Figure 7a shows that mature DP cells from spleen were in R5, indicating that there was a unique DP subset that represents a particular splenic population. These mature DP cells were not significantly altered at $14 \mathrm{dpi}$ with Argentinean CAV strains (Figure 7b). Similar results were obtained for splenic ThL cells, which did not significantly differ between groups. However, the CTL subset decreased only in chickens inoculated with CAV-10 $(p<0.05)$.

\section{Staining with $C D 3 / C D 8 \alpha / C D 8 \beta$}

The G1 region revealed the presence of splenic CD8 lymphocytes, either $\mathrm{CD} 8 \alpha \alpha$ or $\mathrm{CD} 8 \alpha \beta$ cell subsets. The percentages of splenic CD8 $\alpha \alpha$ cells did not significantly differed between groups, although a small increase was observed in CAV-10 inoculated chickens (Figure 8). In contrast, the number of CTL CD $8 \alpha \beta$ was reduced in chickens inoculated with CAV-10 $(p<0.05)$.

\section{Serological testing}

Before inoculation, 3 chickens per group were bled, and all of them were negative to antibodies against CAV. At 14 dpi, seroconversion of animals inoculated with CAV-10 and CAV-18 was monitored. All chickens from CAV-10 and CAV-18 inoculated groups were positive for anti-CAV antibodies ( $\mathrm{S} / \mathrm{N}$ ratio $\leq 0.6)$, whereas birds from the control group remained negative $(\mathrm{S} / \mathrm{N}$ ratio $>0.6)$. At this time post-infection no significant differences in CAV-specific antibody levels could be detected between CAV-10 and CAV-18 inoculated groups (Figure 9).

\section{Discussion}

Complete genome sequencing and comparison of CAV10 and CAV-18 viruses showed few differences between these two Argentinean strains. Both CAV strains have a deletion that contained a fifth 19-nt repeat, with this region being associated with a better efficiency to replicate in MDCC-MSB1 cell cultures [31]. However, previous reports affirmed that the contribution of this region to the attenuation or pathogenicity of CAV in chickens is still questionable [24]. 


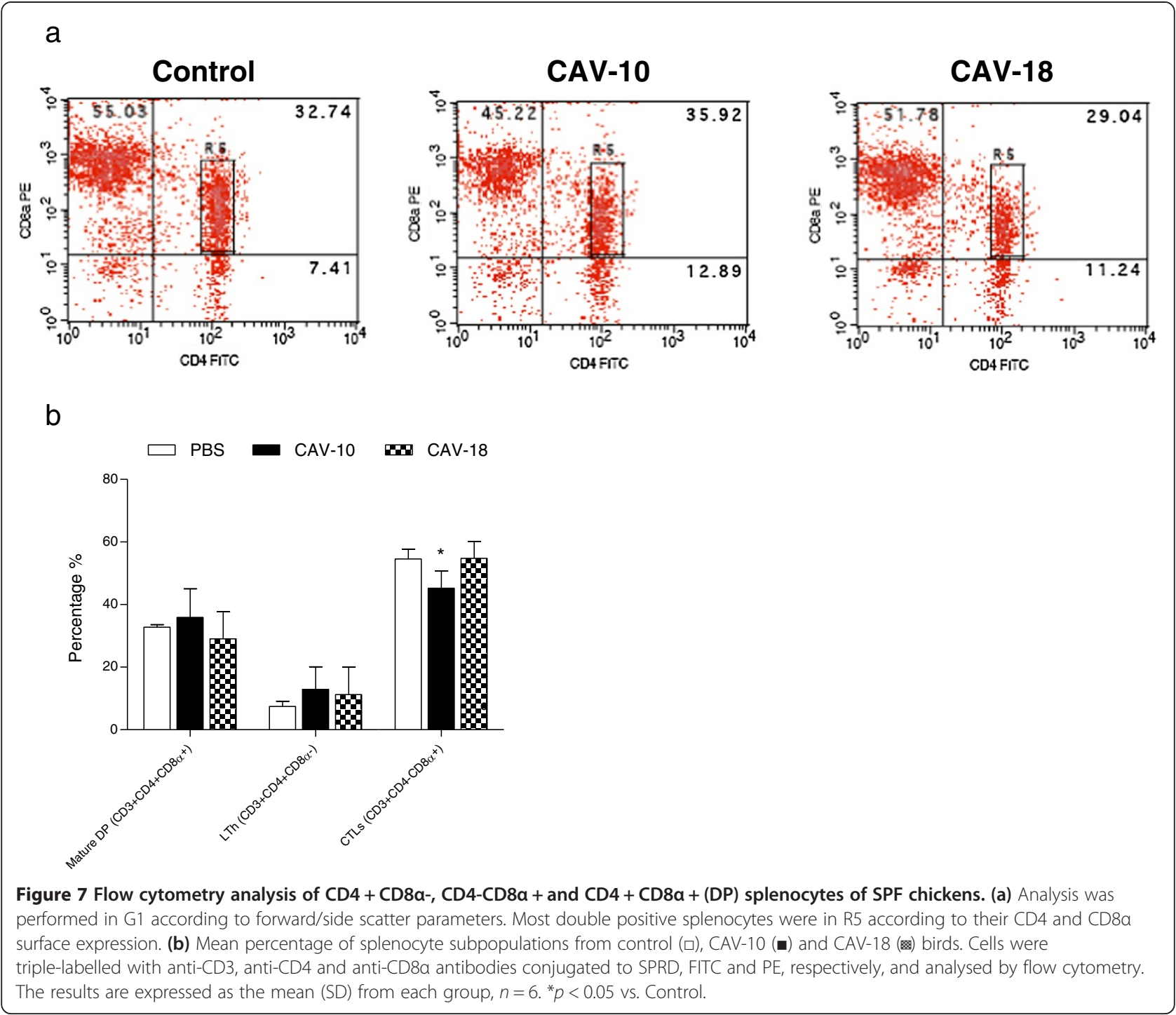

In addition to the deletion, genome sequencing of CAV-10 and CAV-18 confirmed that both viruses present mutations in the hypervariable region of VP1 that are also associated with a reduced spread in an avian lymphoblastoid cell line (MDCC-MSB1) compared with Cux-1 [26]. However, these authors were unable to demonstrate differences in host pathogenesis in vivo due to differences in this region. On the contrary, Meehan et al. [24] have shown that this region does not contribute disproportionately to pathogenicity compared to other regions throughout the genome, and that alterations in more than one of the three CAV proteins, as well as in the noncoding region of the genome, may influence pathogenicity.

Despite the eleven nucleotide differences observed along VP1, only two deduced amino acid substitutions were seen in CAV-10 with respect to the CAV-18 virus. To our knowledge, there are still no reports about the plausible role or the way in which these differences may affect the pathogenesis of CAV in the chicken. We believe that this report could be the first approach to evaluate these amino acid differences of VP1 in vivo, moreover taking into account the few nucleotide variations between CAV-10 and CAV-18 observed only in the non-coding region. However, under our experimental conditions we cannot ensure that the same dosage of each isolate was administered in chickens. Therefore, further studies should be performed to calculate infectivity titres of these field strains in vitro to associate genomic differences between Argentinean CAV strains with different pathogenicities in vivo.

In the present study chickens infected either with CAV-10 or CAV-18 did not present clinical signs of disease throughout the experiment. However, both groups inoculated with Argentinean CAV strains experimented a significant reduction of thymus-to-body weight ratios (CAV-10 produced a significantly higher reduction than 


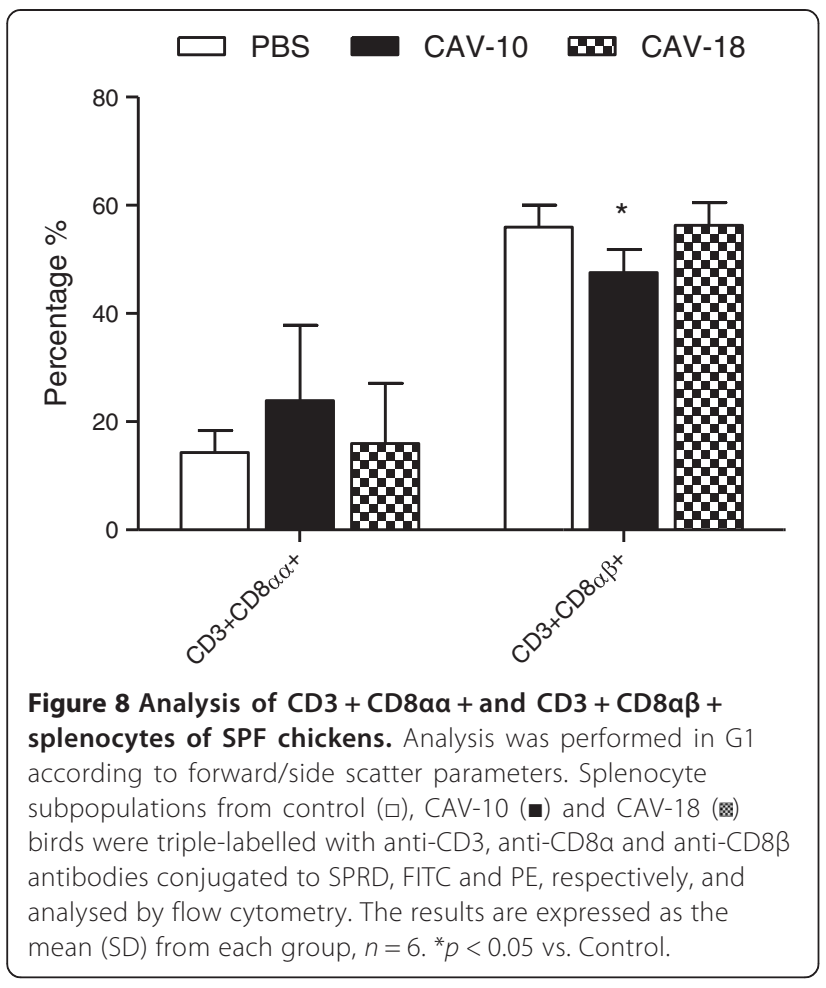

CAV-18) and thymocyte depletion at $14 \mathrm{dpi}$. These results suggest that both CAV field strains produce a subclinical infection in 2-week-old birds.

In addition, chickens infected with CAV-10 virus also show histopathological lesions in the thymus and bursa, compatible with those previously reported by Haridy et al. [32] in subclinical infections with CAV. It is important to mention that these authors infected animals intramuscularly using an isolate that was passaged 10

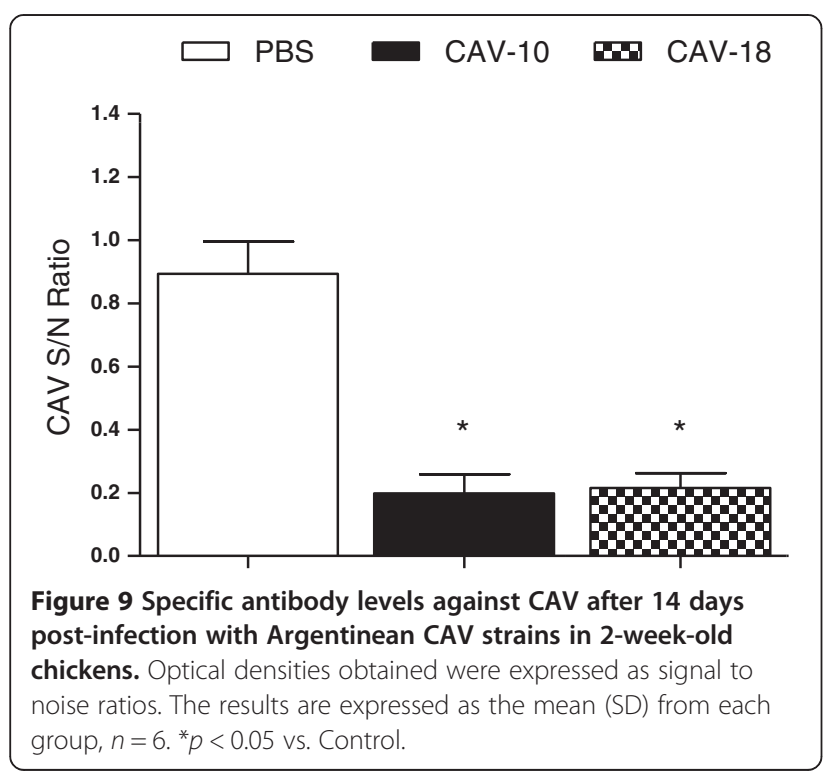

times in MDCC-MSB1 cells [33], where mutations could be introduced. By contrast, CAV-18 inoculated chickens did not present obvious morphological changes in the evaluated organs. This might suggest that alteration of functionality was not very strong or that their course was produced in a very short time in this group and, consequently, the morphological changes could not be observed throughout a histopathological study. Therefore, differences in histopathological lesions produced by CAV-10 or CAV-18 in 2-week-old chickens might suggest that CAV-10 is more aggressive than CAV-18 after in vivo infection, at least at this time post-infection.

Flow cytometry analysis of thymocytes in G1, G2 and G3 from CAV-inoculated chickens vs. control group animals suggests that Argentinean CAV strains alter the thymocyte maturation process after $14 \mathrm{dpi}$.

The most evident variation was the reduction observed in DP thymocytes following CAV inoculation. Similar results were published by Vaziry et al. [21] and $\mathrm{Hu}$ et al. [20], evaluating the lesions in 1-day-old chicks infected with the CIA-1 strain. In addition, our results clearly demonstrate that there are different stages of maturation of DP cells due to thymopoiesis in the thymus, and that some of these stages could be altered after CAV infection. While DP in R4 significantly decreased after CAV infection, DP in R5 significantly increased. These evident changes in the percentages of DP thymocytes with different $C D 4$ and $C D 8 \alpha$ surface expression support the idea that Argentinean CAV viruses can alter thymopoiesis. Even more, under our experimental conditions, CAV-10 produced significantly deeper alterations than CAV-18 in thymocyte maturation two weeks after infection.

On the contrary, the reduction in the total number of lymphocytes in thymuses from CAV inoculated chickens observed during the cell isolation procedure, the absence of significant changes in the number of $\mathrm{CD} 4+\mathrm{CD} 8 \alpha-$ thymocytes and the slight decrease of CD4-CD8 $\alpha+$ cells observed after flow cytometry analysis of the thymic population add to the notion that mature lymphocytes were not recruited into the thymus at 14 dpi with Argentinean CAV strains. These results were in concordance with previous observations by Vaziry et al. [21] in a subclinical infection with a vaccinal strain.

Finally, the alterations in percentages of splenic lymphocyte subpopulations were also analyzed by flow cytometry. There were no differences in mature DP and ThL cells between groups after infection with Argentinean field strains, similar to results previously published by other authors with the CIA-1 vaccinal strain [21]. However, in our experimental infection, CTL cells were statistically reduced in spleens from CAV-10 inoculated chickens. Moreover, staining with $\mathrm{CD} 3, \mathrm{CD} 8 \alpha$ and $\mathrm{CD} 8 \beta \mathrm{mAb}$ allowed discrimination between CD8+ subsets $(C D 8 \alpha \alpha$ or $C D 8 \alpha \beta$ ) that were affected by CAV inoculation. While 
our results show no differences in CD8 $\alpha \alpha$ cells, significant reduction in percentages of the $C D 8 \alpha \beta$ subpopulation was observed only in CAV-10 inoculated birds. Adair et al. [22] have demonstrated the presence of infected mature lymphocytes in the spleen after $6 \mathrm{dpi}$, although the numbers of infected cells as a proportion of the total number recorded was much lower than that in the thymus or bone marrow. Our results, where a significant decrease in splenic $C D 8 \alpha \beta$ lymphocytes was observed at $14 \mathrm{dpi}$, could be the consequence of the large amount of CAV-infected splenocytes reported by Adair et al. at a previous time post-infection. Altogether, our results suggest that CAV-10 affects splenic lymphocyte subpopulations by a reduction in $C D 8 \alpha \beta$ subset and, consequently, this could alter the host immune response against other secondary pathogens.

In this work we studied the subclinical infection of 2-week-old chickens with two Argentinean CAV field strains, showing some nucleotide differences in their genome sequence. Under our experimental conditions, the results might suggest there are differences in the behavior of CAV-10 and CAV-18 strains in vivo at $14 \mathrm{dpi}$. Further studies should be done to determine first the viral titer of these CAV field strains and then, to associate nucleotide/amino acidic composition, in VP1 and/or in noncoding region, with differential pathogenicity in chickens.

\section{List of abbreviations}

CAV: Chicken Anemia Virus; CAV-10: Chicken Anemia Virus, Argentinean strain 10; CAV-18: Chicken Anemia Virus, Argentinean strain 18; CT: Cycle threshold; DP: Double Positive; dpi: Days post-infection; ELISA: Enzyme-Linked ImmunoSorbent Assay; HE: Hematoxylin and eosin stain; MDCC-MSB1: Chicken hematopoietic lymphoblast cell line; ORF: Open Reading Frame;

PCR: Polymerase Chain Reaction; RT-qPCR: Real-time quantitative PCR.

\section{Competing interests}

The authors declare that they have no competing interests.

\section{Authors' contributions}

AR participated in the design of the study and conception, coordinated and carried out the animal studies, the sequence alignment, the cytometry and statistical analysis and drafts the manuscript and revised it critically for important intellectual content. SP carried out the histopathology. VO participated in virus detection, virus sequencing and animal studies. MD participated in animal studies. MPF helped to draft the manuscript. MIC conceived of the study, participated in its design, in animal studies and helped to draft the manuscript. AP conceived of the study, participated in its design, in animal studies and helped to draft the manuscript. AR, SP, VO, MD, MPF, MIC and AP have given final approval of the version to be published.

\section{Acknowledgements \\ The authors thank to Mariela Gammella for acquisition of data by flow cytometry, Juan Manuel Schammas for technical support and María José Gravisaco for interpretation of data. This work was partially supported by the NIAID Center for Research on Influenza Pathogenesis (CRIP) through University of Maryland, College Park, contract no. HHSN266200700010C. This work was also supported by P. E. Enfermedades Infecciosas de las Aves PNSA 1115056 from INTA and by PICT 2008 - 0922 from MINCyT. A. Rimondi is recipient of CONICET fellowship.}

\section{Author details}

'Laboratorio de Aves y Porcinos, Instituto de Virología CICVyA, Instituto Nacional de Tecnología Agropecuaria (INTA), CC25, B1712WAA Castelar,
Buenos Aires, Argentina. ${ }^{2}$ Cátedra de Patología Aviar, Facultad de Ciencias Veterinarias, Universidad de Buenos Aires (UBA), Chorroarín 280, C1427CWO CABA, Argentina. ${ }^{3}$ Consejo Nacional de Investigaciones Científicas y Tecnológicas (CONICET), Rivadavia 1917, C1033AAJ CABA, Argentina.

Received: 28 May 2014 Accepted: 24 September 2014

Published online: 08 October 2014

\section{References}

1. Schat KA: Chicken Infectious Anemia. In Diseases of Poultry. $11^{\text {th }}$ edition. Edited by Saif YM, Barnes HJ, Glisson JR, Fadly AM, McDougald LR, Swayne DE. Ames: lowa State University Press; 2003:182-202.

2. Pringle CR: Virus taxonomy at the XI International Congress of Virology, Sydney, Australia. Arch Virol 1999, 144:2065-2070.

3. Noteborn MHM, Kranenburg O, Zantema A, Koch G, de Boer GF, van der Eb AJ: Transcription of the chicken anemia virus (CAV) genome and synthesis of its 52-kDa protein. Gene 1992, 118:267-271.

4. Phenix KV, Meehan BM, Todd D, McNulty MS: Transcriptional analysis and genome expression of chicken anaemia virus. J Gen Virol 1994, 75:905-909.

5. Koch G, van Roozelaar DJ, Verschueren CAJ, van der Eb AJ, Noteborn MHM: Immunogenic and protective properties of chicken anaemia virus proteins expressed by baculovirus. Vaccine 1995, 13:763-770.

6. Noteborn MHM, Verschueren CA, Koch G, van der Eb AJ: Simultaneous expression of recombinant baculovirus-encoded chicken anemia virus (CAV) proteins VP1 and VP2 is required for formation of the CAV-specific neutralizing epitope. J Gen Virol 1998, 79:3073-3077.

7. Noteborn MH, Todd D, Verschueren CA, de Gauw HW, Curran WL, Veldkamp S, Douglas AJ, McNulty MS, van der Eb AJ, Koch G: A single chicken anemia virus protein induces apoptosis. J Virol 1994, 68:346-351.

8. Jeurissen SHM, Wagenaar F, Pol JMA, van der Eb AJ, Noteborn MHM: Chicken anemia virus causes apoptosis of thymocytes after in vivo infection and of cell lines after in vitro infection. J Virol 1992, 66:7383-7388.

9. McNulty MS: Chicken anemia agent: a review. Avian Pathol 1991, 20:187-203.

10. McNulty MS, Mcllroy SG, Bruce DW, Todd D: Economic effects of subclinical chicken anemia infection in broiler chicks. Avian Dis 1991, 35:263-268.

11. Toro H, Ramirez AM, Laurenas J: Pathogenicity of chicken anemia virus (isolate 10343) for young and older chickens. Avian Pathol 1997, 26:485-499.

12. McConnell CD, Adair BM, McNulty MS: Effects of chicken anemia virus on cell-mediated immune function in chickens exposed to the virus by a natural route. Avian Dis 1993, 37:366-374.

13. Markowski-Grimsrud CJ, Schat KA: Infection with chicken anemia virus impairs the generation of antigen-specific.cytotoxic T lymphocytes. Immunology 2003, 109:283-294.

14. Schat KA: Marek's disease immunosuppression. In Marek's Disease: An Evolving Problem. $1^{\text {st }}$ edition. Edited by Davison F, Nair V. London: Elsevier Academic Press; 2004:49-61.

15. Davidson I, Kedem M, Borochovitz H, Kass N, Ayali G, Hamzani E, Perelman B, Smith B, Perk S: Chicken infectious anemia virus infection in Israeli commercial flocks: virus amplification, clinical signs, performance, and antibody status. Avian Dis 2004, 48:108-118.

16. Zanella A, Dall'Ara P, Lavazza A, Marchi R, Morena MA, Rampin T: Interaction between Marek's disease virus and chicken infectious anemia virus. In Current Progress on Marek's Disease Research. Edited by Schat KA, Morgan RM, Parcells MS, Spencer JL. Kennett Square, PA: American Association of Avian Pathologists; 2001:11-19.

17. Taniguchi T, Yuasa M, Maeda M, Horiuchi T: Chronological observations on hemapathological changes in chicks inoculated with chicken anemia agent. Natl Inst Anim Health Q (Tokyo) 1983, 22:61-69.

18. Goryo M, Hatashi S, Yoshizaida K, Umemura T, Itakura C, Yamashiro S: Ultrastructure of the thymus in chickens inoculated with chicken anemia agent (MSB1-TK 5803 strain). Avian Pathol 1989, 18:605-617.

19. Coud SS, Lillehoj HS, Rosenberger JK: Immune dysfunction following infection with chicken anemia agent and infectious bursal disease virus. I. kinetic alterations of avian lymphocyte subpopulations. Vet Immunol Immunopathol 1992, 34:337-352. 
20. Hu LB, Lucio B, Schat KA: Depletion of CD4+ and CD8+ T lymphocyte subpopulations by $\mathrm{CIA}-1$, a chicken infectious anemia virus. Avian Dis 1993, 37:492-500.

21. Vaziry A, Silim A, Bleau C, Frenette D, Lamontagne L: Chicken infectious anaemia vaccinal strain persists in the spleen and thymus of young chicks and induces thymic lymphoid cell disorders. Avian Pathol 2011, 40:377-385

22. Adair BM, McNeilly F, McConnell CD, McNulty MS: Characterization of surface markers present on cells infected by chicken anemia virus in experimentally infected chickens. Avian Dis 1993, 37:943-950.

23. Drén CN, Kant A, Van Roozelaar DJ, Hartog L, Noteborn MH, Koch G Studies on the pathogenesis of chicken infectious anaemia virus infection in six-week-old SPF chickens. Acta Vet Hung 2000, 48:455-467.

24. Meehan BM, Todd D, Creelan JL, Connor TJ, McNulty MS: Investigation of the attenuation exhibited by a molecularly cloned chicken anemia virus isolate by utilizing a chimeric virus approach. J Virol 1997, 71:8362-8367.

25. Craig MI, Rimondi A, Delamer M, Sansalone P, König G, Vagnozzi A, Pereda A: Molecular characterization of chicken infectious anemia virus circulating in Argentina during 2007. Avian Dis 2009, 53:331-335.

26. Renshaw RW, Soiné C, Weinkle T, O'Connell PH, Ohashi K, Watson S, Lucio B, Harrington S, Schat KA: A hypervariable region in VP1 of chicken infectious anemia virus mediates rate of spread and cell tropism in tissue culture. J Virol 1996, 70:8872-8878.

27. Van Santen VL, Li L, Hoerr FJ, Lauerman LH: Genetic characterization of chicken anemia virus from commercial broiler chickens in Alabama. Avian Dis 2001, 45:373-388.

28. Davidson I, Loeb E, Lublin A, Perk S, Shkoda I, Schat KA: Assessment of various criteria to determine the chicken anemia virus pathogenicity in embryonated eggs and in day-old chicks. Curr Trop Virol 2007, 6:95-111.

29. Technical Reference Promega. [http://www.promega.com/ /media/files/ resources/technical\%20references/plasmid\%20and\%20protein\% 20quantitation.pdf].

30. Noteborn MHM, Verscheuren CAJ, Zantema A, Koch G, van der Eb AJ: Identification of the promoter region of chicken anemia virus (CAV) containing a novel enhancer element. Gene 1994, 150:313-318.

31. Todd D, Connor TJ, Calvert VM, Creelan JL, Meehan BM, McNulty MS: Molecular cloning of an attenuated chicken anaemia virus isolate following repeated cell culture passage. Avian Pathol 1995, 24:171-187.

32. Haridy M, Sasaki J, Ikezawa M, Okada K, Goryo M: Pathological and immunohistochemical studies of subclinical infection of chicken anemia virus in 4-week-old chickens. J Vet Med Sci 2012, 74:757-764.

33. Goryo M, Sugimura H, Matsumoto S, Umemura T, Itakura C: Isolation of an agent inducing chicken anemia. Avian Pathol 1985, 14:483-496.

doi:10.1186/s13567-014-0102-y

Cite this article as: Rimondi et al:: Comparative histopathological and immunological study of two field strains of chicken anemia virus. Veterinary Research 2014 45:102.

\section{Submit your next manuscript to BioMed Central and take full advantage of:}

- Convenient online submission

- Thorough peer review

- No space constraints or color figure charges

- Immediate publication on acceptance

- Inclusion in PubMed, CAS, Scopus and Google Scholar

- Research which is freely available for redistribution 